\title{
Achievable Information Rates for High-Speed Long-Haul Optical Transmission
}

\author{
Ivan B. Djordjevic, Member, IEEE, Bane Vasic, Senior Member, IEEE, Milos Ivkovic, and Ildar Gabitov
}

\begin{abstract}
There have been numerous attempts to determine the channel capacity of a nonlinear fiber-optic communication channel. The main approach was to consider amplified spontaneous emission (ASE) noise as a predominant effect and to observe the fiber nonlinearities as the perturbation of a linear case or as the multiplicative noise. In this paper, the achievable information rates for high-speed optical transmission $(40 \mathrm{~Gb} / \mathrm{s}$ and above) are calculated using the finite-state-machine approach. In calculations, the combined effect of ASE noise, Kerr nonlinearity [selfphase modulation (SPM), intrachannel four-wave mixing (IFWM), intrachannel cross-phase modulation (IXPM)], stimulated Raman scattering (SRS), chromatic dispersion, and (optical/electrical) filtering is taken into account.
\end{abstract}

Index Terms-Achievable information rates, fiber nonlinearities, finite-state machine, long-haul transmission, optical communications, Shannon's capacity.

\section{INTRODUCTION}

$\mathbf{T}$ HE INCREASE of optical bandwidth and improvement of spectral efficiency are common ways to increase the throughput of a dense wavelength division multiplexing (DWDM) system. Recent progress in DWDM transmission technology has led to a fundamental and a very difficult question: "What is the Shannon's capacity of a nonlinear fiber-optic communication channel?" There have been numerous attempts to tackle this problem [1]-[13]. The common approach is to consider the amplified spontaneous emission (ASE) from inline amplifiers as a predominant effect and include the effect of nonlinearities in an approximate fashion. The fiber nonlinearities are considered either as 1) the perturbation of a linear case [4], [12], or as 2) the multiplicative noise [6].

Although the perturbative methods, in general, may yield reliable results in the domain of validity [4], they are applicable in the regime of relatively small nonlinearities, which is not applicable for high-speed transmission (at $40 \mathrm{~Gb} / \mathrm{s}$ or higher) systems. Mitra and Stark [6] treat a nonlinear noisy channel as a linear one with an effective nonlinear noise. However, as indicated in [2], such an approximation needs to be carefully justified for each particular transmission system. In dispersionfree transmission [7], [8], the nonlinear Schrödinger equation

Manuscript received December 27, 2004; revised May 10, 2005. This work was supported in part by the National Science Foundation (NSF) under Grants ITR 0325979 and CCR 0208597.

I. B. Djordjevic and B. Vasic are with the Department of Electrical and Computer Engineering, University of Arizona, Tucson, AZ 85719 USA (e-mail: ivan@ece.arizona.edu; vasic@ece.arizona.edu).

M. Ivkovic and I. Gabitov are with the Department of Mathematics, University of Arizona, Tucson, AZ 85721 USA (e-mail: milos@math.arizona. edu; gabitov@math.arizona.edu).

Digital Object Identifier 10.1109/JLT.2005.857751
(NLSE) [14] can be solved analytically, but such a result is only of academic interest [7]. In [3], Tang determined the channel capacity of a multispan DWDM system employing dispersive nonlinear optical fibers and an ideal coherent optical receiver. The results obtained in [3] are based on solving the NLSE by Volterra series expansion up to the first order. Such a method is valid in systems for which the maximum nonlinear rotation is small compared to $2 \pi$ [3]. The channel capacity is determined using Pinsker's formula [15], which may lead to wrong conclusions, as it was shown in [2], especially for high signal-to-noise ratios. The statistics of optical transmission in a noisy nonlinear channel with weak dispersion management and zero average dispersion is considered in [2], and the lower bounds for channel capacity are determined (although numerical results are not reported). However, this method is applicable only for weak dispersion management systems with zero average dispersion, as described in [16]. Some papers, e.g., [13], completely ignore the influence of dispersion and nonlinearities. The spectralefficiency limits in DWDM systems with coherent detection in nonlinear regime limited independently by cross-phase modulation (XPM) or four-wave mixing (FWM) are reported in [1]. As opposed to $10-\mathrm{Gb} / \mathrm{s}$ systems, where the major nonlinearities are due to interchannel effects, in $40 \mathrm{~Gb} / \mathrm{s}$ and above, the major nonlinearities are due to intrachannel nonlinearities, such as intrachannel FWM (IFWM), intrachannel XPM (IXPM), and intrachannel self-phase modulation (SPM). Therefore, the weak nonlinearity assumption is not applicable, and the results on channel capacity are valid only if the combined effects of ASE noise, Kerr nonlinearities, dispersion, and filtering effects are taken into account in calculations.

In this paper, we report the achievable information rates (lower bounds on channel capacity) for high-speed long-haul optical transmission with an independent uniformly distributed (i.u.d.) source, when the combined effect of ASE noise, Kerr nonlinearity (SPM, IFWM, IXPM), stimulated Raman scattering (SRS), chromatic dispersion [group velocity dispersion (GVD), second-order GVD], and (optical/electrical) filtering is taken into account. The method employs a finite-state-machine approach outlined in [17]-[20], and it was initially proposed for magnetic channels, which are essentially nonlinear as well. The propagation of the signal is modeled by the NLSE, which is solved using the Fourier split step algorithm [14]. The ASE noise-signal interaction during transmission is taken into account, and the fiber channel is modeled as a channel with memory. The proposed approach is applicable to any transmission system and allows the usage of the achievable information rate (similarly as $Q$-factor, eye opening, or bit-error rate) as a figure of merit for a long-haul transmission system. 


\section{Calculation of Achievable Information Rates USING Finite-STATE-MACHINE APPROACH}

The method used to determine a lower bound on the achievable information rate, which is proposed here, is to estimate the mutual information between the input random process $X$ and output process $Y$ by modeling the channel as a finitestate machine [17]-[20]. The finite-state machine is described by the input alphabet $X$, output alphabet $Y$, finite set of states $S$, and by the conditional probability density function, $p\left(y_{n}, s \mid s^{\prime}\right) \quad\left(y_{n} \in Y ; s\right.$ - the current state, $s^{\prime}$ - the previous state), determined by simulation. It is assumed that $m$ previous and $m$ next bits influence the observed bit, and the state $s=\left(x_{n-m}, x_{n-m+1}, \ldots, x_{n}, x_{n+1}, \ldots, x_{n+m}\right) \in S$ is determined by a sequence of $2 m+1$ input bits $x_{i} \in X=\{0,1\}$.

The problem of computing channel capacity $C$ involves the maximization of mutual information $I(X, Y)$ over all possible input distributions $p(X)$

$$
C=\max _{p(X)} I(X, Y)
$$

and the mutual information is defined as

$$
I(X, Y)=H(Y)-H(Y \mid X)
$$

with $H(Y)=-E[\log p(Y)]$ being the output-process entropy rate. According to the Shannon-McMillan-Breimann theorem [18], [19], for a stationary ergodic finite-state Markov process $Y$

$$
-\frac{1}{n} \log p\left(Y^{n}\right) \rightarrow H(Y)
$$

as $n \rightarrow \infty$, where $p\left(Y^{n}\right)=\operatorname{Prob}\left[\left(Y_{1}, Y_{2}, \ldots, Y_{n}\right)\right]$. So, the information rate (1) can be estimated from a long output sequence $y^{n}=\left(y_{1}, y_{2}, \ldots, y_{n}\right)$ by calculating $\log p\left(y^{n}\right)$.

As already mentioned, we reduce our attention to the i.u.d. source (the case commonly considered in practice), and therefore, to the calculation of the achievable information rate [19], which is a lower bound for the channel capacity.

The $\log p\left(y^{n}\right)$ for an i.u.d. source can be estimated by the Bahl-Cocke-Jelinek-Raviv (BCJR) algorithm [21]

$$
\log p\left(y^{n}\right)=\sum_{i=1}^{n} \log p\left(y_{i} \mid y^{i-1}\right)
$$

where

$$
p\left(y_{i} \mid y^{i-1}\right)=\sum_{s^{\prime}, s} \alpha_{i-1}\left(s^{\prime}\right) P\left(y_{i} \mid s\right) P_{s^{\prime} s} .
$$

The probability $\alpha_{i}(s)$ of the state $s$ at instance $i$ is calculated by

$$
\alpha_{i}(s)=\frac{\sum_{s^{\prime}} \alpha_{i-1}\left(s^{\prime}\right) P\left(y_{i} \mid s\right) P_{s^{\prime} s}}{\sum_{s^{\prime}, s} \alpha_{i-1}\left(s^{\prime}\right) P\left(y_{i} \mid s\right) P_{s^{\prime} s}}
$$

where $P_{s^{\prime} s}$ is the probability of transition from state $s^{\prime}$ to state $s$, and the conditional probability $P\left(y_{i} \mid s\right)$ is estimated from simulations.
The conditional entropy $H(Y \mid X)$ is calculated simply as

$$
\lim _{n \rightarrow \infty}\left[-\frac{1}{n} \sum_{i=1}^{n} \log P\left(y_{i} \mid s\right)\right]
$$

where $s=\left(x_{n-m}, x_{n-m+1}, \ldots, x_{n}, x_{n+1}, \ldots, x_{n+m}\right) \in S$.

\section{System Model Description}

The signal channel transmission at high bit rate $(40 \mathrm{~Gb} / \mathrm{s}$ and above) is considered with the channel located at $1552.524 \mathrm{~nm}(193.1 \mathrm{THz})$. The carrier-suppressed return-tozero (CSRZ) modulator, which is employed in simulations, is composed of a laser diode, two Mach-Zehnder (MZ) intensity modulators (the first serving as modulator, the second as a nonreturn to zero (NRZ)-to-RZ converter), and a pseudorandom bit sequence (PRBS) generator. The direct detection receiver observed is composed of an optical filter (of bandwidth $2 R_{\mathrm{b}}$; $R_{\mathrm{b}}$ - bit rate), a p-i-n photodiode, an electrical filter (of bandwidth $0.65 R_{\mathrm{b}}$ ), and a sampler followed by a decision circuit. An erbium-doped fiber amplifier (EDFA) is used as a preamplifier.

The dispersion map, shown in Fig. 1, is composed of $N$ spans of length $L$, consisting of $L_{1} \mathrm{~km}$ of $D_{+}$fiber followed by $L_{2} \mathrm{~km}$ of $D_{-}$fiber, with precompensation of $-320 \mathrm{ps} / \mathrm{nm}$ and corresponding postcompensation. The fiber parameters are given in Table I. Three dispersion maps are considered: 1) $L=48 \mathrm{~km}$ or $96 \mathrm{~km}$, and $L_{1}=2 L / 3$;2) $L=48 \mathrm{~km}$ and $L_{1}=2 L / 3$ with no pre- and postcompensation being applied; and 3) $L=47.12 \mathrm{~km}$ and $L_{1}=L / 1.178$, with $D_{+}$and $D_{-}$ fiber sections being replaced by conventional single-mode fiber (SMF) and dispersion-compensating fiber (DCF) with parameters given in Table I. To compensate for the fiber loss, EDFAs, with the noise figure (NF) of $6 \mathrm{~dB}$ in Figs. 5-7 and $5 \mathrm{~dB}$ in Figs. 8-10, are located after every fiber section.

For the light propagation through the fiber, the NLSE was solved using the split-step Fourier method [14]. The effects of Kerr nonlinearities (SPM, IXPM, IFWM), SRS, dispersion (GVD, second-order GVD), and intersymbol interference were taken into account.

\section{NumericAl RESUlTS}

The algorithm to calculate the achievable information rate, which was proposed in Section II, may be summarized into two steps:

1) determination of the conditional probabilities $p\left(y_{i} \mid s\right)$ and the transition probabilities $P_{s^{\prime}, s}$ by propagating a sufficiently long sequence through the transmission system described in Section III, and finding output for different noise realizations;

2) calculation of the output entropy rate $H(Y)$ by using (3)-(5) and calculation of conditional entropy $H(Y \mid X)$ using (6).

The algorithm is general and applicable to both the channels with memory and the memoryless channels. As an illustration, Fig. 2 shows histograms obtained for memoryless additive white Gaussian noise (AWGN) ON-OFF keying (OOK) [Fig. 2(a)] and fiber-optic channels [Fig. 2(b), (c)], after 20 spans of map 1) described in Section III, with launched 


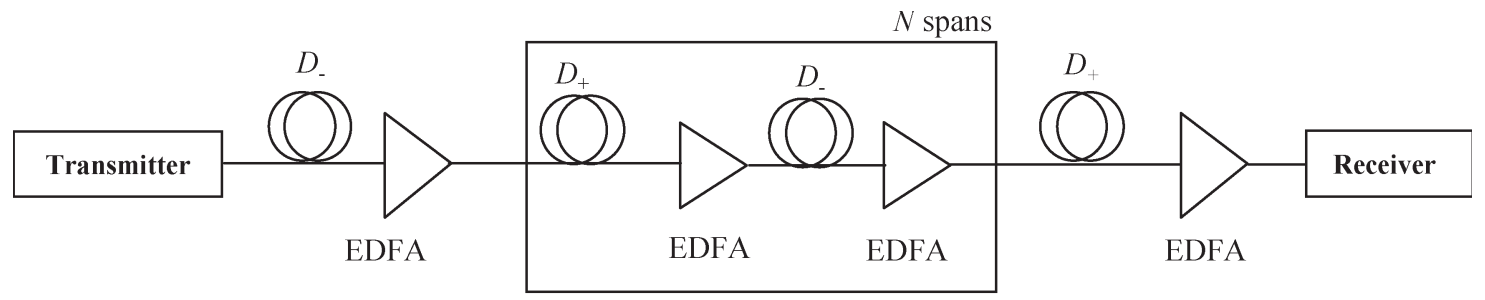

(a)

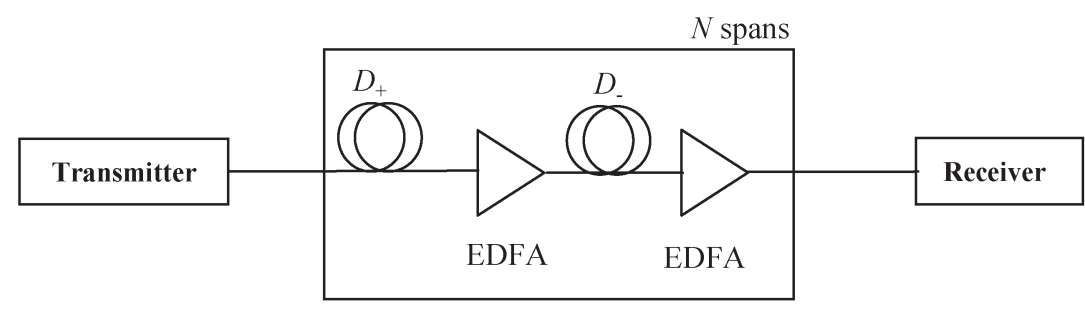

(b)

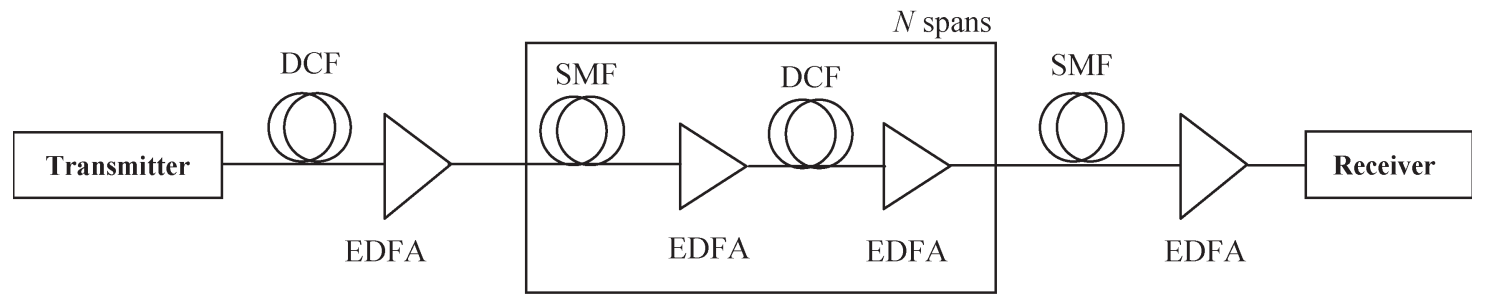

(c)

Fig. 1. Dispersion maps under study: (a) dispersion map with dispersion pre- and postcompensation, (b) dispersion map without dispersion pre- and postcompensation, (c) dispersion map based on standard SMF.

TABLE I

FIBER PARAMETERS

\begin{tabular}{ccccc}
\hline & D + FIBER & D. FIBER & SMF & DCF \\
\hline $\begin{array}{c}\text { Dispersion } \\
{[\mathrm{ps} /(\mathrm{nm} \mathrm{km})]}\end{array}$ & 20 & -40 & 16 & -90 \\
\hline $\begin{array}{c}\text { Dispersion Slope } \\
{\left[\mathrm{ps} /\left(\mathrm{nm}^{2} \mathrm{~km}\right)\right]}\end{array}$ & 0.06 & -0.12 & 0.08 & -0.45 \\
\hline $\begin{array}{c}\text { Effective Cross-sectional Area } \\
{\left[\mu \mathrm{m}^{2}\right]}\end{array}$ & 110 & & & \\
\hline $\begin{array}{c}\text { Nonlinear refractive index } \\
{\left[\mathrm{m}^{2} / \mathrm{W}\right]}\end{array}$ & $2.6 \cdot 10^{-20}$ & $2.6 \cdot 10^{-20}$ & & \\
\hline Attenuation Coefficient & & & 20 & \\
{$[\mathrm{~dB} / \mathrm{km}]$} & 0.19 & 0.25 & & \\
\hline
\end{tabular}

power of $6 \mathrm{dBm}$. Two extreme cases are included, namely an isolated "one" [Fig. 2(d)], and a "zero" symbol surrounded by all "ones" [Fig. 2(c)]. The length of a sequence is $2^{15}-1$, and 16 samples per bit and 64 bins per histogram are used. It is obvious that the commonly used AWGN distribution is not a good model in this case. In Fig. 3(a), the algorithm proposed in Section II is compared against the semianalytical approach we proposed in [11] for the AWGN OOK channel model, and an excellent agreement is found. Fig. 3(b) compares the asymmetric AWGN channel and chi-square channel models. Notice that other models, such as [1] and [3]-[13], are essentially the channel models without memory. Moreover, the channel is modeled as a continuous-input continuous-output channel and the discrete nature of the transmitted information is 


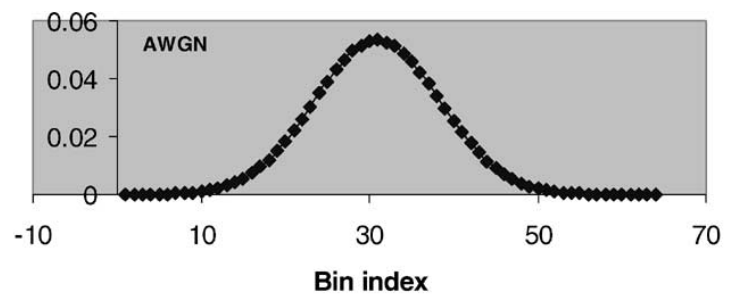

(a)

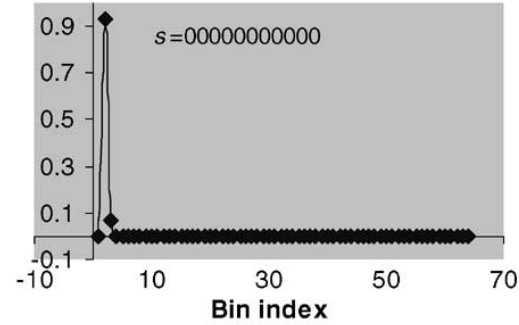

(b)

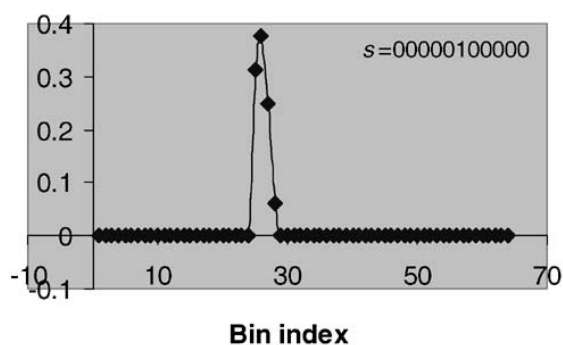

(d)

Fig. 2. Simulator histograms.

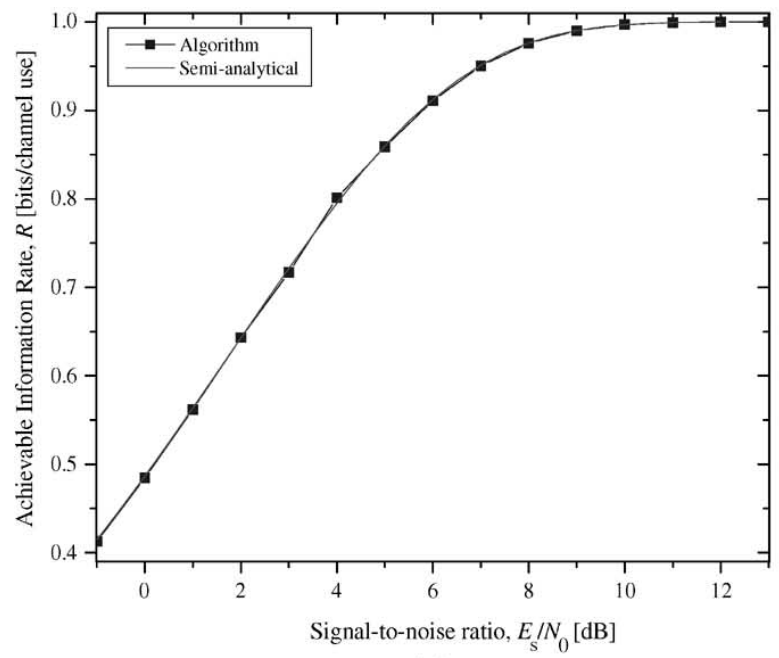

(a)

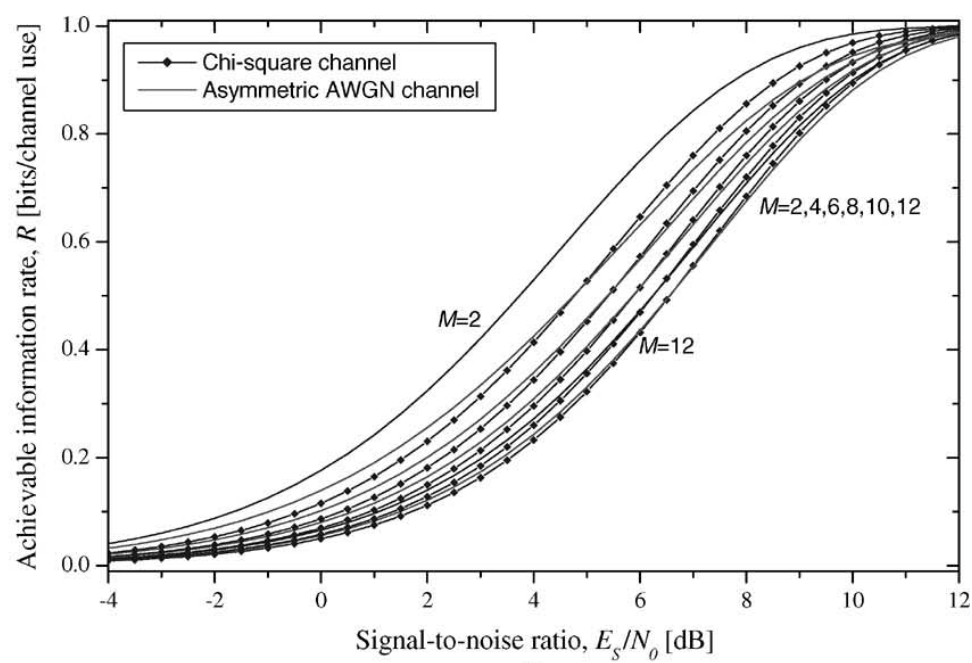

(b)

Fig. 3. Achievable information rates of memoryless channels. (a) Algorithm proposed in Section II against the semianalytical approach from [11] for the AWGN OOK channel model (with 64 bins per histogram and sequence length $\left.n=10^{6}\right)$. (b) Chi-square channel against asymmetric AWGN channel $(M$ denotes the optical-bandwidth-to-electrical-bandwidth ratio).

ignored completely. To be more specific, let us briefly analyze the model by Tang [3] as an illustrative example. The channel capacity of a multispan DWDM system for dispersive nonlinear optical fiber and an ideal coherent optical receiver is analyzed. It is assumed that 1) NLSE can be solved approximately by using Volterra series expansion up to the first order, and that 2) Pinker's formalism is applicable. Neither of these two as- sumptions is valid for data rates of $40 \mathrm{~Gb} / \mathrm{s}$ or higher because the interaction of ASE noise and signal during transmission cannot be ignored, and the influence of XPM, FWM, and SRS must be included. The only nonlinear effect taken into account was the SPM, and it was assumed that SPM is weak. Moreover, Pinker's formalism is applicable to continuous-input continuous-output linear channels [22] only, and may lead to 


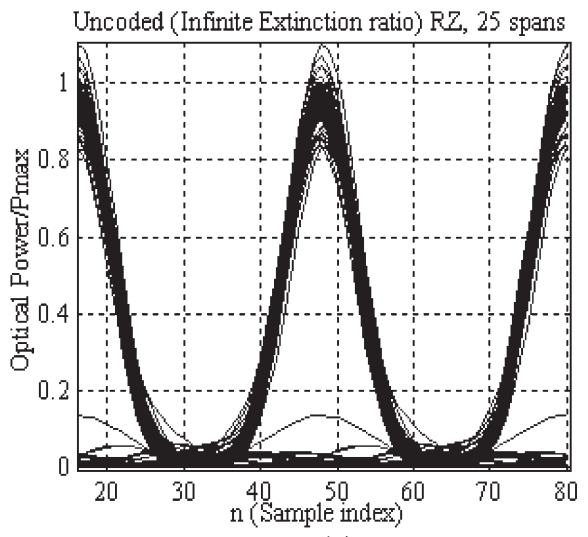

(a)

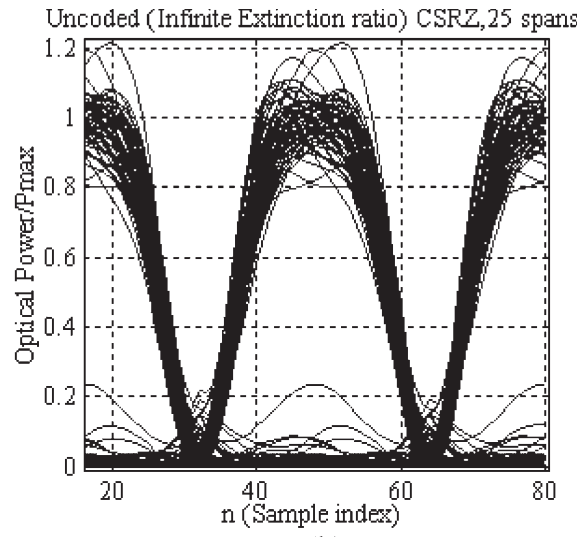

(b)

Fig. 4. Eye diagrams after 25 spans of dispersion map 1) for infinite extinction ratio. (a) RZ. (b) CSRZ.

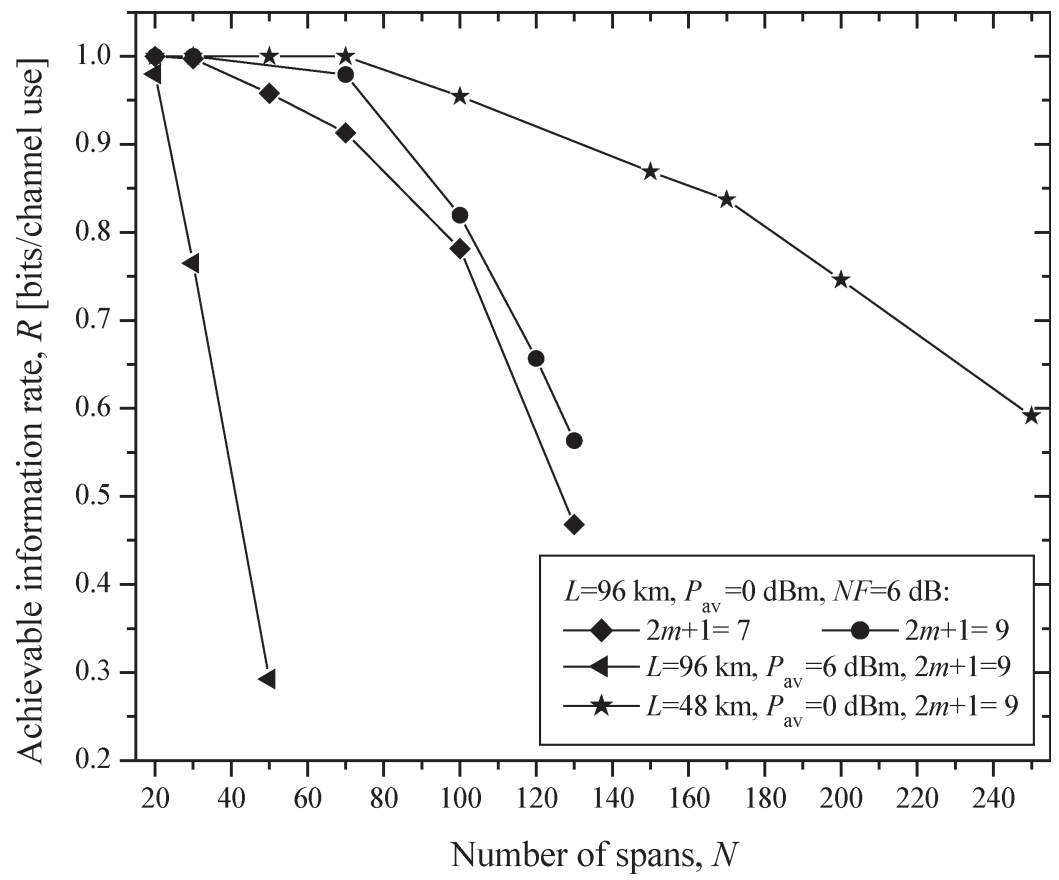

Fig. 5. Achievable information rate versus number of spans for two different map strengths and two different span lengths at $40 \mathrm{~Gb} / \mathrm{s}$ (the number of bins is set to 10$)$.

wrong conclusions, as shown in [2]. In the dispersion map considered in [3], the influence of the DCF part was ignored, and the channel memory was ignored as well.

In the rest of this section, the results obtained employing the algorithm from Section II are discussed. As demonstrated in our recent publications [23], [24], the simulator used for generating the histograms gives an excellent agreement with commercially available VPItransmissionMaker WDM version 5.5. In Fig. 4, the eye diagrams after 25 spans of RZ and CSRZ, for dispersion map 1), are shown as the illustration. The amplitude jitter and ghost pulse effects due to IFWM, and the timing jitter due to IXPM may easily be identified, suggesting that the simulator is able to catch all important nonlinear effects.

To determine the achievable information rates, the PRBS sequence of length $2^{17}-1$, with 32 samples per bit, is transmitted ten times over the whole transmission system for different ASE-noise ensembles; the corresponding conditional probabilities were estimated and fed to the algorithm described in Section II. The sequence length $n$ in (2)-(6) is set to $10^{6}$. The results of simulations are given in Figs. 5-7 for the dispersion map 1). As expected, for larger channel memory and longer sequence of bits, better estimates for the information rate are obtained. For a particular dispersion map, there exists a region of optimum average launched powers (Fig. 6), which is the behavior not expected from the analytical model presented in [1], where a single value for optimum average launched power is predicted. It is also interesting (see Fig. 7) that there exists an optimum bit rate (at around $35 \mathrm{~Gb} / \mathrm{s}$, for number of bins set to 10). It is important to notice that the finite-state machine can efficiently capture all nonlinear effects one would like to include in the model as long as they have local character, i.e., the energy transfer between distant symbols is limited. 


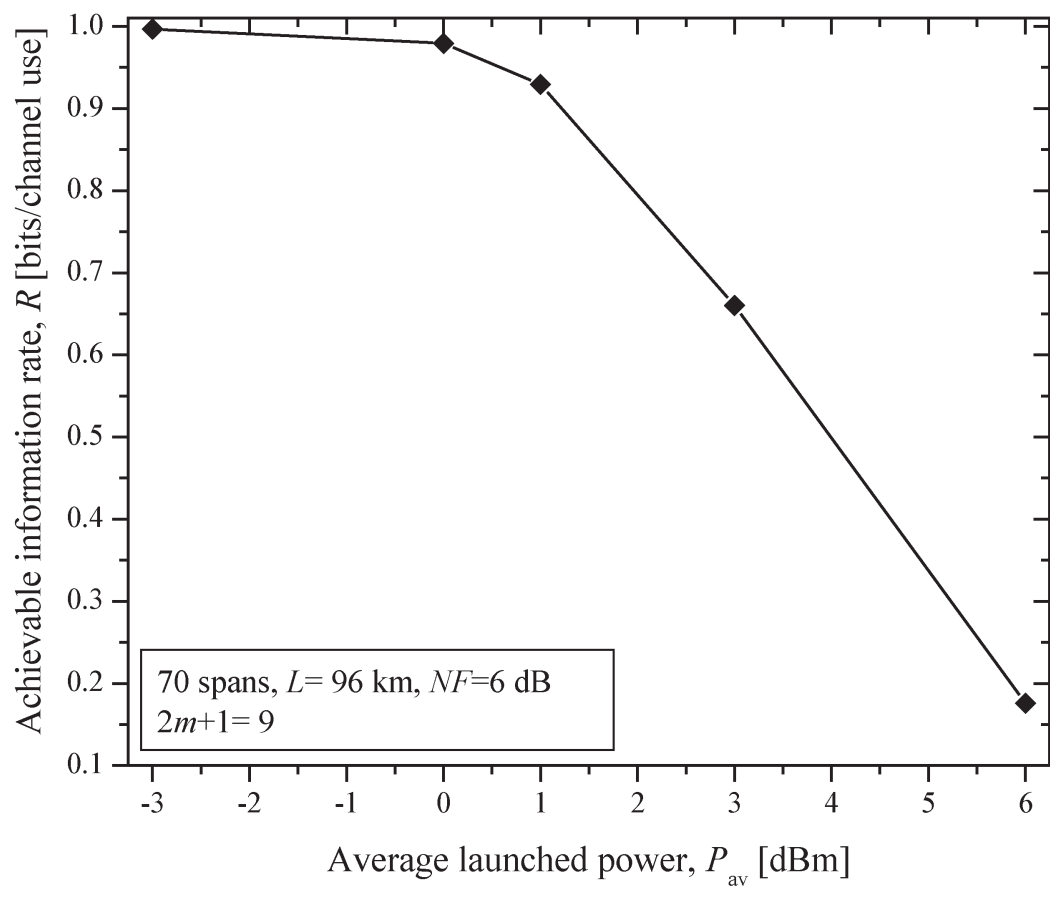

Fig. 6. Achievable information rate versus averaged launched power at $40 \mathrm{~Gb} / \mathrm{s}$ (the number of bins is set to 10).

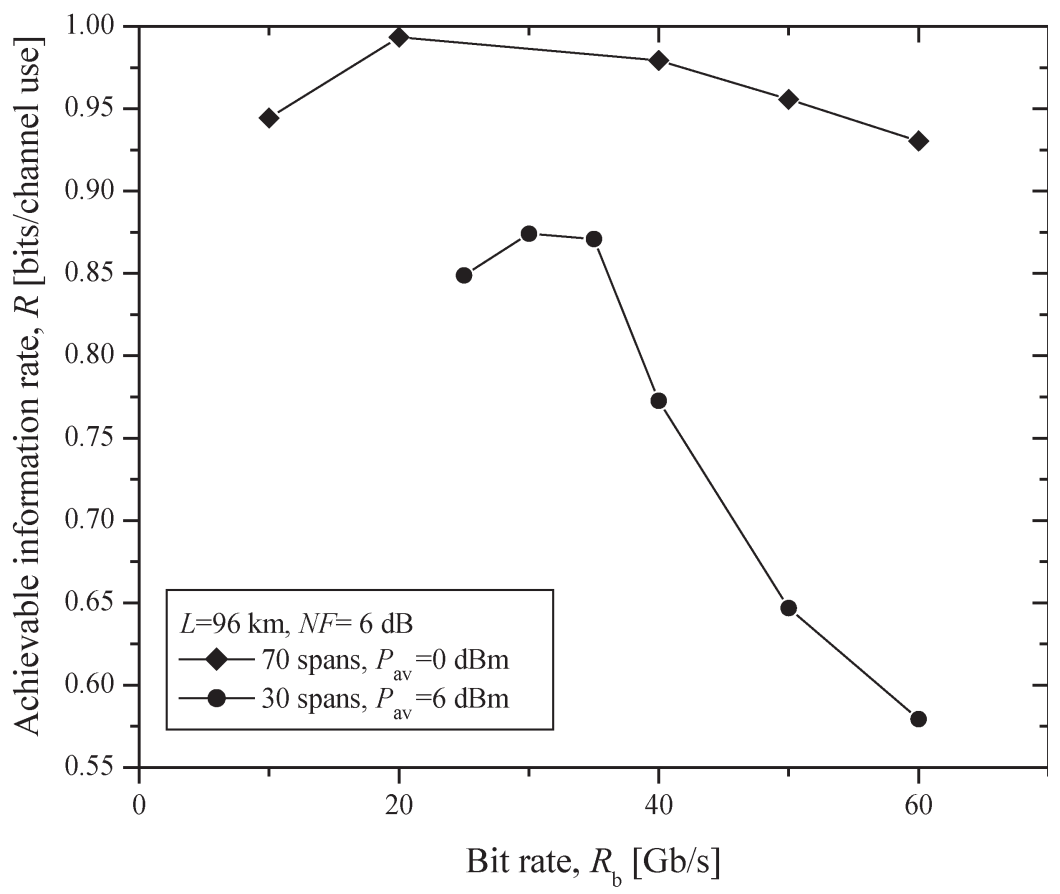

Fig. 7. Achievable information rate versus bit rate (the number of bins is set to 10).

To study the importance of ASE noise-signal interaction during transmission on the Shannon's-capacity calculation, the simulation in which PRBS sequence of length $2^{15}-1$ is transmitted once over different dispersion maps and equivalent ASE noise added at the end of transmission system is performed for 1000 ASE-noise ensembles, and results are shown in Figs. 9 and 10(a) for different channel memories. Similar dependences as in Fig. 5 are obtained; however, the achievable information rates are grossly underestimated. The simulations in which ASE noise-signal interaction during transmission is ignored may only be used in qualitative comparison of different dispersion maps with respect to the channel capacity; but a complete picture can only be obtained by computationally extensive simulations where the ASE noise-signal interaction (during transmission) is included. Considerations in which the optical channel is modeled as a perturbed AWGN channel are, therefore, rather questionable. From Figs. 8-10, it is evident that the achievable information rate depends very strongly 


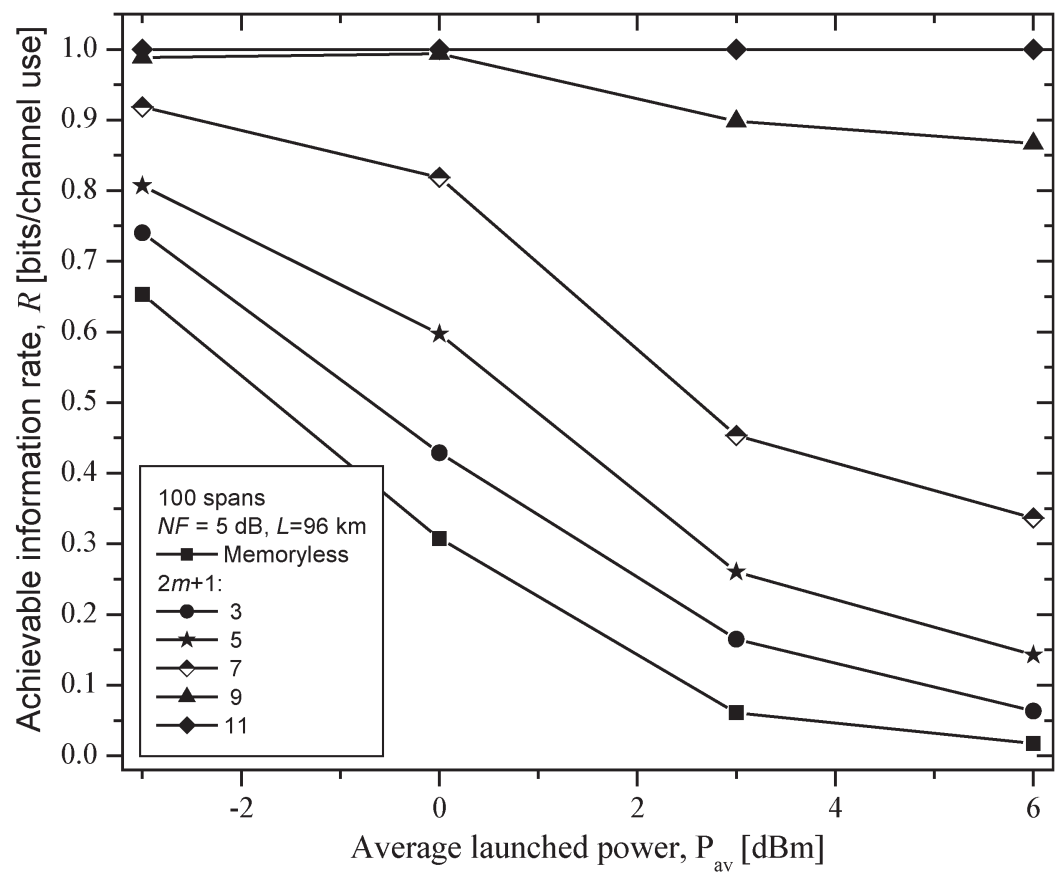

Fig. 8. Achievable information rate versus averaged launched power at $40 \mathrm{~Gb} / \mathrm{s}$ for different channel memories (the number of bins is set to 128).

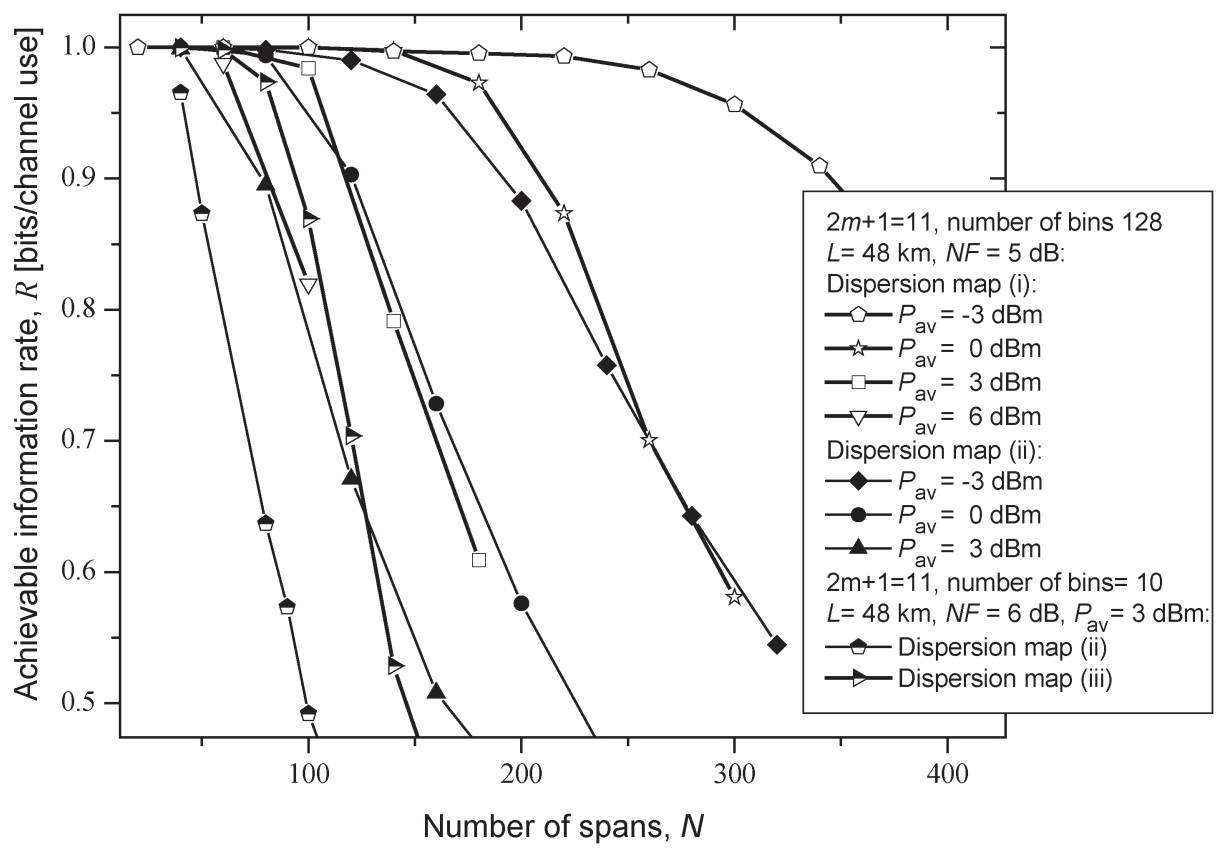

Fig. 9. Achievable information rate versus number of spans for three different dispersion maps at $40 \mathrm{~Gb} / \mathrm{s}$, when ASE noise-signal interaction during transmission was ignored.

on the channel-memory assumptions. The memoryless-channel assumption, commonly used in literature, may lead to quite incorrect conclusions on channel capacity. If the number of bins used in determination of histograms is too small, the channel capacity is also underestimated.

The importance of this finding is profound in two ways. First, it clearly demonstrates the inaccuracy of models that do not take the channel memory into account. Such models underestimate the achievable information rate because they treat the intersymbol interference due to dispersion and non- linearities as a random process, whereas it is, in fact, a deterministic phenomenon that can be, in principle, compensated in the receiver. Second, it shows that the existing optical communication systems are highly underutilized because the state-of-the-art detection algorithms try to cancel the intersymbol interference instead of using it as a means to effectively improve the performance. The design of such a receiver is a complex problem, and is beyond the scope of this paper (the first step in this direction was made in our recent paper [25]). 


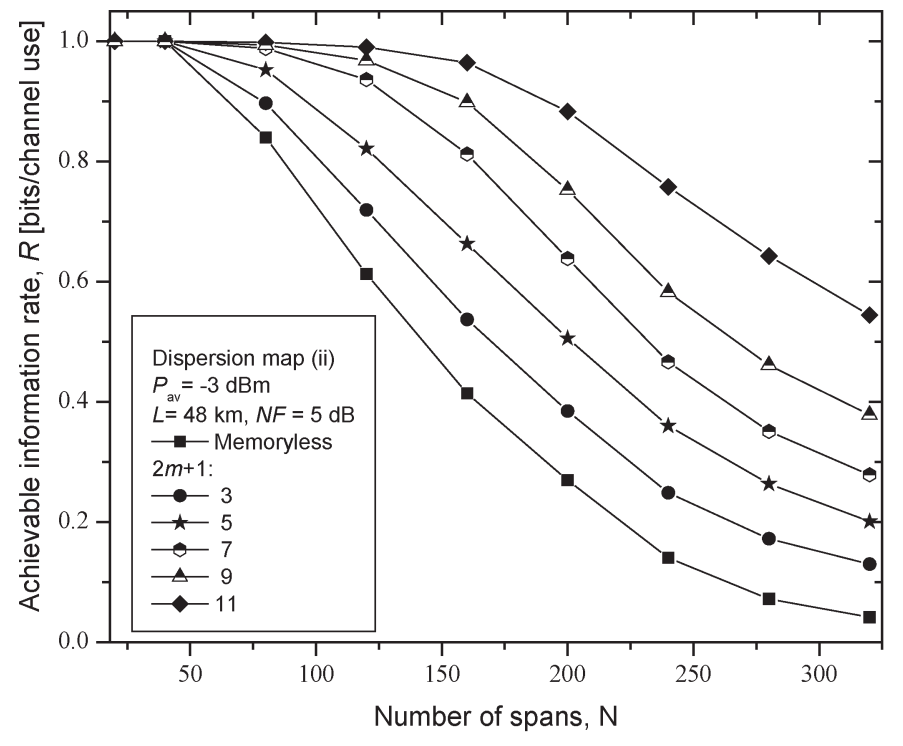

(a)

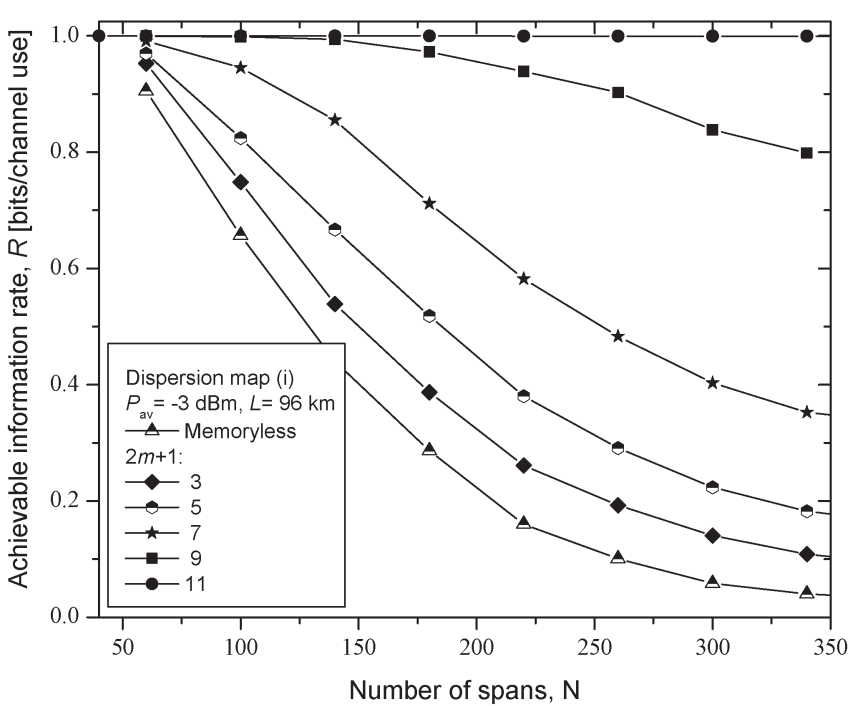

(b)

Fig. 10. Achievable information rate versus number of spans for different channel memories at $40 \mathrm{~Gb} / \mathrm{s}$. (a) Dispersion map 3) with ASE noise-signal interaction during transmission being ignored (span length set to $48 \mathrm{~km}$ ). (b) Dispersion map 1) with signal-noise interaction during transmission being included (span length set to $96 \mathrm{~km}$; the number of bins is set to 128 ).

\section{CONCLUSION}

Achievable information rates for high-speed optical transmission (40 Gb/s and above) are calculated using finite-statemachine approach. The combined effect of all major effects including: ASE noise, Kerr nonlinearity (SPM, IFWM, IXPM), SRS, chromatic dispersion (GVD, second-order GVD), and (optical/electrical) filtering on the Shannon's capacity is considered. The lower bound for the channel capacity is determined for various dispersion maps and number of spans, and shown to depend strongly on the dispersion map and average launched power. The user bit rate maximizing the spectral efficiency is found to be around $35 \mathrm{~Gb} / \mathrm{s}$, for number of bins set to 10 .

It is important to notice that the proposed approach is applicable to any nonlinear system, including the optical recording, magnetic recording, free-space optics, and biological ones, as long as the system can be modeled using the finite-statemachine approach.

\section{REFERENCES}

[1] J. M. Kahn and K.-P. Ho, "Spectral efficiency limits and modulation/ detection techniques for DWDM systems," IEEE J. Sel. Topics Quantum Electron., vol. 10, no. 2, pp. 259-272, Mar./Apr. 2004.

[2] K. S. Turitsyn, S. A. Derevyanko, I. V. Yurkevich, and S. K. Turitsyn, "Information capacity of optical fiber channels with zero average dispersion," Phys. Rev. Lett., vol. 91, no. 20, p. 203901, Nov. 2003.

[3] J. Tang, "The channel capacity of a multispan DWDM system employing dispersive nonlinear optical fibers and an ideal coherent optical receiver," J. Lightw. Technol., vol. 20, no. 7, pp. 1095-1101, Jul. 2002.

[4] E. E. Narimanov and P. Mitra, "The channel capacity of a fiber optics communication system: Perturbation theory," J. Lightw. Technol., vol. 20, no. 3, pp. 530-537, Mar. 2002.

[5] A. Mecozzi and M. Shtaif, "On the capacity of intensity modulated systems using optical amplifiers," IEEE Photon. Technol. Lett., vol. 13, no. 9, pp. 1029-1031, Sep. 2001.

[6] P. P. Mitra and J. B. Stark, "Nonlinear limits to the information capacity of optical fiber communications," Nature, vol. 411, no. 6841, pp. 10271030, Jun. 2001.
[7] J. Tang, "The Shannon channel capacity of dispersion-free nonlinear optical fiber transmission," J. Lightw. Technol., vol. 19, no. 8, pp. 1104-1109, Aug. 2001.

[8] - "The multispan effects of Kerr nonlinearity and amplifier noises on Shannon channel capacity for a dispersion-free nonlinear optical fiber," J. Lightw. Technol., vol. 19, no. 8, pp. 1110-1115, Aug. 2001.

[9] K.-P. Ho and J. M. Kahn, "Channel capacity of WDM systems using constant-intensity modulation formats," in Proc. Optical Fiber Соттиnication (OFC), Anaheim, CA, 2002, pp. 731-733, Paper ThGG85.

[10] J. M. Kahn and K.-P. Ho, "Ultimate spectral efficiency limits in DWDM systems," in Proc. Optoelectronics Communications Conf., Yokohama, Japan, Jul. 8-12, 2002, pp. 552-553.

[11] I. B. Djordjevic and B. Vasic, "Approaching Shannon's capacity limits of fiber optics communications channels using short LDPC codes," presented at the Conf. Lasers and Electro-Optics/Int. Quantum Electronics Conf. (CLEO/IQEC), San Francisco, CA, Paper CWA7.

[12] E. Narimanov and P. Patel, "Channel capacity of fiber optics communications systems: WDM vs. TDM," in Proc. Conf. Lasers and ElectroOptics (CLEO), Baltimore, MD, 2003, pp. 1666-1668.

[13] J. Li, "On the achievable information rate of asymmetric optical fiber channels with amplifier spontaneous emission noise," in Proc. IEEE Military Communications Conf. (MILCOM), Boston, MA, Oct. 2003, pp. 124-129.

[14] G. P. Agrawal, Nonlinear Fiber Optics. San Diego, CA: Academic, 2001.

[15] M. S. Pinsker, Information and Information Stability of Random Variables and Processes. San Francisco, CA: Holden Day, 1964.

[16] S. K. Turritsyn et al., "Averaged model and integrable limits in nonlinear double-periodic Hamiltonian systems," Phys. Rev. E, vol. 61, no. 3, pp. 3127-3132, Mar. 2000.

[17] D. Arnold, A. Kavcic, H.-A. Loeliger, P. O. Vontobel, and W. Zeng, "Simulation-based computation of information rates: Upper and lower bounds," in Proc. IEEE Int. Symp. Information Theory (ISIT), Yokohama, Japan, 2003, p. 119.

[18] D. Arnold and H.-A. Loeliger, "On the information rate of binary-input channels with memory," in Proc. Int. Conf. Communications, Helsinki, Finland, Jun. 11-14, 2001, pp. 2692-2695.

[19] H. D. Pfitser, J. B. Soriaga, and P. H. Siegel, "On the achievable information rates of finite state ISI channels," in Proc. Global Telecommunications (GLOBECOM), San Antonio, TX, Nov. 25-29, 2001, pp. 2992-2996.

[20] V. Sharma and S. K. Singh, "Entropy and channel capacity in the regenerative setup with applications to Markov channels," in Proc. IEEE Int. Symp. Information Theory, Washington, DC, Jun. 24-29, 2001, p. 283. 
[21] L. R. Bahl, J. Cocke, F. Jelinek, and J. Raviv, "Optimal decoding of linear codes for minimizing symbol error rate," IEEE Trans. Inf. Theory, vol. IT-20, no. 2, pp. 284-287, Mar. 1974.

[22] J. G. Proakis, Digital Communications. Boston, MA: McGraw-Hill, 2001.

[23] B. Vasic, V. Rao, I. B. Djordjevic, R. Kostuk, and I. Gabitov, "Ghost pulse reduction in $40 \mathrm{~Gb} / \mathrm{s}$ systems using line coding," IEEE Photon. Technol. Lett., vol. 16, no. 7, pp. 1784-1786, Jul. 2004.

[24] I. B. Djordjevic, S. K. Chilappagari, and B. Vasic, "Suppression of intrachannel nonlinear effects using pseudo-ternary constrained codes," J. Lightw. Technol., to be published.

[25] B. Vasic, I. B. Djordjevic, and V. S. Rao, "Advanced detection and coding techniques for nonlinear intersymbol interference cancellation in $40 \mathrm{~Gb} / \mathrm{s}$ systems," Inst. Elect. Eng.-Optoelectron., submitted for publication.

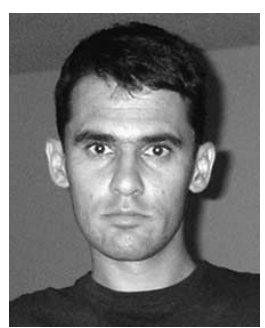

Ivan B. Djordjevic (M'04) received the B.Sc., M.Sc., and Ph.D. degrees, all in electrical engineering, from the University of Nis, Serbia, in 1994, 1997 , and 1999 , respectively.

From 1994 to 1996, he was with the University of Nis, and from 1996 to 2000, he was with the State Telecommunications Company (Serbia Telecom), District Office for Networks-Nis. He was involved in digital transmission systems commissioning and acceptance, design, maintenance, installation, and connection. From 2000 to 2001, he was with the National Technical University of Athens, Greece, and with TyCom U.S. Inc. (now TyCo Telecommunications). He was involved in modelling and simulation of WMD systems and networks. During 2002 and 2003, he was with the University of Arizona, Tucson; University of Bristol, U.K.; and University of the West of England, Bristol; working on forward error correction and iterative decoding for optical transmission, optical CDMA, high-speed transmission, and optical switches. He is now with the University of Arizona, on leave from the University of the West of England. He is the author of more than 100 international journal articles and international conference papers. His research interests include DWDM fiber-optic communication systems and networks, error control coding, OFDM, free space optics, CDMA, optical packet switching, coherent communications, statistical communication theory, and satellite and wireless communications.

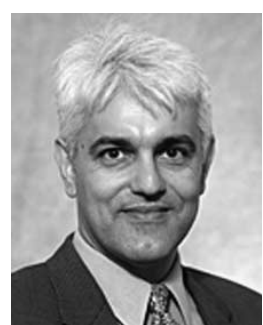

Bane Vasic (S'92-M'93-SM'02) received the B.Sc., M.Sc., and $\mathrm{Ph} . \mathrm{D}$. degrees, all in electrical engineering, from the University of Nis, Serbia, in 1990, 1991, and 1994, respectively.

From 1996 to 1997, he worked as a Visiting Scientist at the Rochester Institute of Technology and Kodak Research, Rochester, NY, where he was involved in research in optical storage channels. From 1998 to 2000, he was with Lucent Technologies, Bell Laboratories. He was involved in research coding schemes and architectures for high-speed applications. He was involved in research in iterative decoding and low-density parity check codes, as well as development of codes and detectors implemented in Lucent (now Agere) chips. Presently, he is a Faculty Member of the Electrical and Computer Engineering Department, University of Arizona, Tucson. His research interests include coding theory, information theory, communication theory, and digital communications and recording.

Dr. Vasic is a member of the Editorial Board of the IEEE TRANSACTIONS ON MAGnETiCs. He served as a Technical Program Chair, IEEE Communication Theory Workshop, 2003, and as Co-organizer of the Center for Discrete Mathematics and Theoretical Computer Science (DIMACS) Workshops on Optical/Magnetic Recording and Optical Transmission, and Theoretical Advances in Information Recording, 2004. He is a co-organizer of the Communication Theory Symposium within the International Conference on Communications (ICC 2006).

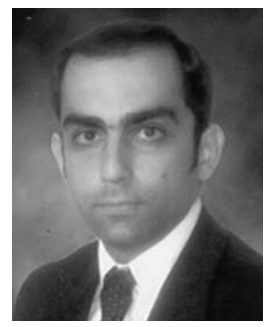

Milos Ivkovic graduated in mathematics from the University of Belgrade, Serbia, in 2000 and received the Master's degree in applied mathematics from the State University of Campinas (UNICAMP), Brazil, in 2002. He is currently pursuing the Ph.D. degree at the University of Arizona, Tucson.

Ildar Gabitov, photograph and biography not available at the time of publication. 